During the past decade a number of studies have examined the effect of prophylactic lithium treatment on the mortality and suicidal behaviour of manic-depressive patients. Two reviews, Tondo et al (1997) and Schou (1998), have analysed them and found a statistically significant association between long-term lithium treatment, on the one hand, and reduced mortality and suicidal behaviour on the other. According to the first review, the number of suicidal acts was on average 3.2 per 100 patient-years in patients without lithium and 0.37 per 100 patient-years in patients with lithium, an 8.2-fold difference. The second review showed that whereas manic-depressive patients have a mortality that is two to three times that in the general population, the mortality of patients with lithium treatment was not higher or was only slightly higher than in the general population. The number of suicide attempts was 6-15 times lower and the number of completed suicides 3-17 times lower when the patients were on lithium than when they were not. When lithium treatment was discontinued, the mortality and the suicidal behaviour increased.

These observations indicate strongly that prophylactic lithium treatment has an anti-suicidal action, but correlation is not the same as causation, and other factors than the treatment may have played a role. It is, however, noteworthy that an association between suicidal behaviour and drug treatment has not been demonstrated for use of other mood stabilisers, where the same factors must have been at work. In a European, randomised, collaborative longterm trial, no suicidal act occurred in patients on lithium, whereas nine suicidal acts were observed in patients having received carbamazepine (Thies-Flechtner et al, 1996).

The findings reviewed above do not prove definitely that lithium has an antisuicidal action but the evidence does not reject such a hypothesis. We suggest that prophylactic lithium treatment should be given to manic-depressive patients at high risk of committing suicide, that is patients with severe depression or depression with persistent suicidal ideation or with a history of suicide attempts.

Isacsecon, G., Holmgren, P., Druld, H., et of (1999) Psychotropics and suicide prevention. Implications from toxicological screening of 5281 suicides in Sweden 1992-1994. British journol of Psychiatry 174, 259-265.

Schou, M. (1998) The effect of prophylactic lithium treatment on mortality and suicidal behavior: A review for clinicians. Journal of Affective Disorders, 50, 253-259.
Thies-Flechtner, K., Muller-Oerlinghausen, B., Seibert, W., et al (1996) Effect of prophylactic lithium treatment on suicide risk in patients with affective disorders: Data from a randomized prospective study. Pharmacopsychiatry, 29, 103-107.

Tondo, L., Jamison, K. R. Baldessarini, R. U. (1997) Effect of lithium maintenance on suicidal behavior in major mood disorders. Annals of the New York Academy of Sciences, 836, 339-351.

B. Müller-Oerlinghausen Department of

Psychiatry, Research Group of Clinical

Psychopharmacology, Freie Universität Berlin,

Eschenallee 3, 14050 Berlin, Germany

P. Grof Royal Ottawa Hospital, Health Care Group, I145 Carling Avenue, Ottawa, Ontario,

Canada KIZ7 K4

M. Schou Department of Biological Psychiatry, Aarhus University Hospital, Skovagervej 2, 8240 Risskov, Denmark

\section{Alcohol, hypertension and cognitive decline}

Sir: Stewart (1999) highlights some interesting issues regarding the role of hypertension in dementia. No reference is made, however, to the potential role of alcohol in these conditions. Alcohol excess has been linked independently to both cognitive impairment and high blood pressure, although the concept of a distinct primary alcoholic dementia remains controversial (Smith \& Atkinson, 1995).

Stewart observes that an apparent fall in blood pressure can occur at the onset or during the development of dementia. This process may well be related to alcohol withdrawal in some individuals. One of the pathways into abstinence seen in older alcoholics occurs when some consequences of the dementing process (e.g. institutionalisation) limit the supply of drink and propel the individual into an 'involuntary' abstinence. In such cases the patient may also experience a fall in blood pressure as alcohol is withdrawn, contributing to the clinical picture described by Stewart. Curtis et al (1997) have documented alterations in systolic blood pressure which correlate with changes in drinking status. The role of alcohol should be investigated in future longitudinal studies of hypertension.

Curtis, A. B., James, S. A., Strogatz, D. S., et al (1997) Alcohol consumption and changes in blood pressure among African Americans: The Pitt county study. American journal of Epidemiology. 146, 727-733.

Smith D. M. \& Atkinson, R. M. (1995) Alcoholism and dementia. International journal of Addictions, 30. 1843-1869.
Stewart, R. (1999) Hypertension and cognitive decline. British journal of Psychiatry, 174, 286-287.

1. Johnson Division of Psychiatry, University of Bristol, 4I St Michael's Hill, Bristol BS2 8DZ

\section{Prolonged seizures detectable by electroencephalogram in electroconvulsive therapy}

Sir: Mayur et al (1999) reported that 38 $(16.4 \%)$ of 232 patients experienced prolonged cerebral seizure activity detectable by two-channel electroencephalogram (EEG) at the first treatment in a course of electroconvulsive therapy (ECT). In contrast, Abrams (1997) argued that the existing evidence suggested prolonged cerebral seizure activity is rare in the routine practice of ECT, and is usually associated with coexisting brain disease. This debate prompted us to re-analyse the findings of two Scottish studies previously published in the Journal to report for the first time the prevalence of prolonged cerebral seizure activity in UK practice.

The Royal College of Psychiatrists (1995) recommended that seizures that last longer than 120 seconds ought to be regarded as prolonged and be terminated. The American Psychiatric Association recommended a cut-off of 180 seconds (American Psychiatric Association, 1990).

In the Dumfries study, single-channel EEG monitoring was conducted at most treatments given to 41 patients (McCreadie et al, 1989). Usable EEG recordings were obtained at 263 treatments (101 unilateral treatments and 162 bilateral treatments). One 23-year-old woman experienced prolonged seizures at her first four bilateral treatments in a course of six treatments. One 55-year-old woman experienced a prolonged seizure at her first bilateral treatment only. The prevalence of prolonged seizure activity at the first treatment was 2 out of 41 patients $(4.9 \%, 95 \% \mathrm{CI}-1.7$ to $11.5 \%$ ) for seizures longer than 120 seconds, and 1 out of 41 patients $(2.4 \%, 95 \%$ CI -2.3 to $7.1 \%$ ) for seizures longer than 180 seconds.

In the Edinburgh study, six-channel EEG monitoring was carried out at the outset and end of treatment plus a variable number of intermediate treatments in 22 patients (Scott et al, 1989). Usable EEG recordings were available for 100 treatments (43 unilateral treatments and 57 bilateral treatments). No prolonged seizures were detected. 